\title{
Direct Electron Transfer Anisotropy of a Site-Specifically Immobilized Cellobiose Dehydrogenase
}

Su Ma ${ }^{1}$, Christophe V.F.P Laurent ${ }^{1,2}$, Marta Meneghello ${ }^{3}$, Jani Tuoriniemi ${ }^{4}$, Chris Oostenbrink $^{2,}$ Lo Gorton ${ }^{4}$, Philip N. Bartlett ${ }^{3}$, Roland Ludwig ${ }^{1 *}$

${ }^{1}$ Biocatalysis and Biosensing Laboratory, Department of Food Science and Technology, BOKU - University of Natural Resources and Life Sciences, Muthgasse 18, 1190 Vienna, Austria

${ }^{2}$ Institute of Molecular Modeling and Simulation, BOKU - University of Natural Resources and Life Sciences, Muthgasse 18, 1190 Vienna, Austria

${ }^{3}$ School of Chemistry, Faculty of Engineering and Physical Sciences, University of Southampton, Southampton, SO17 1BJ, UK

${ }^{4}$ Department of Analytical Chemistry/Biochemistry and Structural Biology, Lund University, P.O.Box 124, SE-221 00 Lund, Sweden

* Corresponding author, e-mail: roland.ludwig@boku.ac.at 


\begin{abstract}
To study the direct electron transfer (DET) of the multi-cofactor enzyme cellobiose dehydrogenase $(\mathrm{CDH})$ in regard to its orientation on an electrode surface, a recently published, maleimide-based immobilization method was used in combination with sitedirected mutagenesis to establish different orientations on an electrode surface. CDH from Myriococcum thermophilum was chosen for this study, because its protein structure is resolved and the factors influencing the movement of its mobile cytochrome domain (CYT) are established. Seven CDH variants with a surface exposed cysteine residue in different spatial positions were generated for site-specific maleimide coupling. Surface plasmon resonance and cyclic voltammetry showed that all CDH variants, but not the wild-type $\mathrm{CDH}$, bound covalently to gold electrodes or glassy carbon electrodes and were catalytically active. For DET, the CYT domain needs to move from the closed-state conformation, where it obtains an electron from the catalytic FAD cofactor to the open state where it can donate an electron to the electrode. We therefore hypothesized that the mobility of the CYT domain and its distance to the electrode is central for DET. We found that the uniform spatial orientations of CDH influenced DET as follows: an orientation of the two-domain enzyme on the side, with CYT in proximity to the electrode resulted in high DET currents. Orientations with a bigger distance between CYT and the electrode, or orientations where CYT could not swing back to the dehydrogenase domain (DH) to form the closed enzyme conformation, reduced DET. In the latter case calcium ions, that stabilize the closed conformation of $\mathrm{CDH}$, fully recovered DET. The study demonstrates, that a mobile CYT domain can compensate unfavorable orientations of the catalytic domain to a great extent and allows $\mathrm{CDH}$ as a multi-cofactor enzyme to transfer electrons even in awkward orientations. The mobile CYT domain reduces the anisotropy of DET, which is also essential for CDH's physiological function as an extracellular, electron transferring enzyme.
\end{abstract}

Keywords: cellobiose dehydrogenase, direct electron transfer, site-specific mutagenesis, molecular modelling, anisotropic orientation, site-specific immobilization 


\section{Introduction}

Direct electron transfer (DET) between a redox-active protein and an electrode depends on several factors such as a suitable protein fold, which determines the position and accessibility of the redox cofactor and the electron transfer path, the potential difference between the cofactor and the electrode and the binding of the protein molecule onto the electrode surface. ${ }^{1}$ The orientation of the bound oxidoreductase defines the electron transfer distance of individual protein molecules and thereby the electron transfer (ET) rate. Only oxidoreductases exhibiting efficient DET can be used as electrode catalysts for mediator-less biosensors and biofuel cells. The position of the catalytic cofactor within the protein fold is fine-tuned to the position of the active-site and fixed in place by binding and ligating amino acids and therefore only in some cases, e.g. laccase (EC 1.10.3.2), ${ }^{2}$ horseradish peroxidase $(1.11 .1 .7)^{3}$ or Ni-Fe hydrogenase (EC 1.12.2.1), ${ }^{4}$ able to perform efficient DET. In nature, oxidoreductases that evolved towards ET to other proteins typically carry an additional cytochrome subunit or cytochrome domain for that purpose. These multi-cofactor, DET-capable oxidoreductases combine catalytic cofactors such as molybdenum (sulfite oxidase, EC 1.8.3.1), iron-sulfur clusters (nitrate reductase, EC 1.7.5.1), pyrrolinoquinoline quinone (pyranose dehydrogenase, EC 1.1.2.B5) and FAD (lactate dehydrogenase, EC 1.1.2.3) with an electron transferring cytochrome. ${ }^{5}$ In this case, the distance between the electrode surface and the cytochrome determines the DET rate.

The orientation of an enzyme on an electrode depends on the electrode material, electrode surface modification and the immobilization conditions. The orientation more or less random and influenced by the interaction of the protein surface with the electrode surface. ${ }^{6}$ The so-called natural orientation of an enzyme on a particular electrode is directed by the preferred interaction of surface patches of charged, hydrophobic or hydrophilic amino acids. Adsorption without specific chemical binding has been exploited using different electrode surface modifications such as phenyl derivatives, ${ }^{7}$ azines, ${ }^{8}$ pyrene pyrrole, ${ }^{9}$ or thiols with different head group functionalities $\left(-\mathrm{OH},-\mathrm{NH}_{2},-\mathrm{COOH}\right) .{ }^{10}$ However, the protein orientation achieved by targeting physical properties of surface amino acids via adsorption or undirected covalent immobilization is difficult to predict and hard to control. As an alternative, the sitespecific immobilization of enzymes on electrodes has been introduced to strictly control the orientation of enzymes. One strategy is to use affinity tags like the His-tag, ${ }^{11}$ but this allows only immobilization in two positions: at the $\mathrm{N}$-terminus and the C-terminus. 
Site-directed mutagenesis can be used for the modification of every surface amino acid and therefore any enzyme orientation on the electrode. Examples are the incorporation of the unnatural amino acid 4-azido-L-phenylalanine in a small laccase, ${ }^{12}$ or the substitution of surface amino acids by cysteine in horseradish peroxidase. ${ }^{13}$

Since DET of cellobiose dehydrogenase (CDH, EC 1.1.99.18) on thiol modified gold electrodes was first demonstrated in 2000, ${ }^{14}$ many studies focused on its enhancement. $\mathrm{CDH}$ is a multi-cofactor enzyme and is composed of a catalytic, FAD containing dehydrogenase domain (DH) and an electron transferring, heme $b$ containing cytochrome domain (CYT). ${ }^{15}$ Its diverse substrate spectrum (cellobiose, lactose and glucose) and robust DET make CDH highly interesting for biosensor and biofuel cell applications. ${ }^{16}$ Approaches to increase the DET current of $\mathrm{CDH}$ focused mainly on electrode surface modifications to promote the CYT-electrode interaction by modulating surface hydrophobicity and electrostatics. Different head group functionalities $\left(-\mathrm{NH}_{2},-\mathrm{COOH}\right.$ and $\left.-\mathrm{OH}\right)$ were featured by the modification of gold electrodes with thiol-based self-assembled monolayers (SAM). All tested CDHs gave highest electrocatalytic currents on SAMs with -OH head groups. ${ }^{17}$ SAMs exhibiting a $-\mathrm{COOH}$ head group resulted in lower current densities because of the electrostatic repulsion between the negatively charged CYT domain (pI $~ 3)$ and - $\mathrm{COOH}$ modified SAMs. For glassy carbon/single walled carbon nanotube electrodes that are modified with $p$-aminophenyl groups the positive surface charge resultes in a much larger current than that measured at a $p$-aminobenzoic acid-modified negatively charged surface. ${ }^{18}$ Recently, a covalent, site-specific immobilization method for $\mathrm{CDH}$ was reported, ${ }^{19}$ which allows the anisotropic orientation of $\mathrm{CDH}$ by the position of a cysteine introduced to the protein surface. The reported method also allows the adjustment of the protein packing density at electrode surface.

To study the effect of oriented immobilization of CDH on DET, we employed Myriococcum thermophilum (syn. Crassicarpon hotsonii, MtCDH) for two reasons: Firstly, it has a resolved crystal structure (PDB ID: 4QI6), which allows the structure guided selection of amino acids for site-directed mutagenesis and subsequent sitespecific immobilization. Secondly, the possibility to modulate the CYT domain conformation in $\mathrm{MtCDH}$ between the closed-state and the open-state by switching $\mathrm{pH}$ and $\mathrm{Ca}^{2+}$ ion concentration can be used to investigate the CYT mobility for different orientations. The enhancement of the DET current of $M t C D H$ by $\mathrm{Ca}^{2+}$ ions was reported by Schulz and Gorton ${ }^{20}$ and followed by several studies on the effect of divalent cations. 
The catalytic current of $\mathrm{MtCDH}$ at $\mathrm{pH} 5.5$ was increased 5.1-fold when adding $50 \mathrm{mM}$ $\mathrm{CaCl}_{2}$ and the apparent reduction rate of CYT was increased 10.4-fold, indicating a faster interdomain electron transfer (IET) ${ }^{20}$ Hydrogen/deuterium exchange (HDX-MS) experiments revealed that IET of CDH is governed by surface electrostatics and positive charged calcium ions bridge the negative charges of the domain interfaces. ${ }^{21}$ The crystal structure of CDHs confirmed the open and closed conformation of CDH and indicated a significant role of the propionate-interacting residues in $\mathrm{DH} .{ }^{15} \mathrm{~A}$ further structural investigation of CDH by small angle X-ray scattering and small angle neutron scattering showed that $M t C D H$ undergoes conformational rearrangement in the presence of calcium ions. $^{22}$

To test our hypothesis that the free movement of CYT and its distance to the electrode are the factors for fast electron transfer, we selected surface exposed amino acids in different regions of the DH domain (top, bottom, front, back, left and right side) to be mutated into cysteines serving as anchors for site-specific immobilization. A specific reaction of the free thiol group of cysteine and maleimide-modified electrodes was used to covalently bind the enzyme to the electrode. This resulted in anisotropically oriented CDHs with a limited rotational freedom. The immobilization of CDH variants on the electrode surface was monitored by surface plasmon resonance (SPR) and cyclic voltammetry (CV) was employed to study the role of CYT as built-in redox mediator of the flavocytochrome CDH.

\section{Results and discussion}

1. Site-specific introduction of surface cysteines

We used structure-based site-specific engineering to generate $\mathrm{CDH}$ variants with a single, surface exposed Cys residue that can be covalently linked to an electrode surface and keep the enzyme in a specific spatial orientation (Table S1). Seven individual positions on the back, front, side, top, and bottom of the DH domain were chosen, and are indicated as subscripts for easy identification (Figure 1). The numbering of the amino acids follows the reported crystal structure of MtCDH (PDB ID: 4QI6) and excludes the 21 aa signal sequence. Mutation D792C $\mathrm{C}_{\text {top }}$ on CDH's carbohydrate binding module (CBM) results after covalent immobilization onto the electrode in a top-on orientation, which is the natural orientation of $\mathrm{CDH}$ on cellulose. The inverse

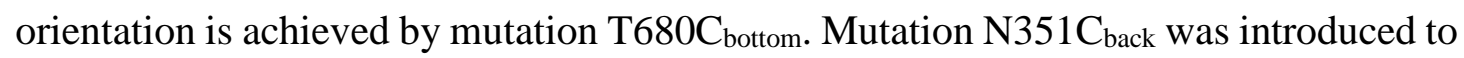


obtain the farthest distance between the heme cofactor of CYT and the electrode. An orientation with DH's substrate channel facing the electrode is difficult to realize, because CYT also interacts with $\mathrm{DH}$ at the substrate channel in the closed conformation to accept an electron from the catalytic FAD cofactor. Therefore, residue E501, which is in proximity of the protein interface but does not interfere with the interaction of the domains was selected. Two mutations were introduced on the left side of the substrate

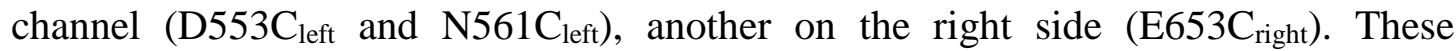
introduced anchor residues are located within structurally stable regions, such as an $\alpha$ helix or a small loop, to reduce distortion or unfolding of the protein scaffold (Figure S1). The solvent-exposed orientation of the side-chains of the selected residues is necessary to enable the coupling reaction between the cysteine's thiol group and the electrode-attached maleimide group.

\section{Recombinant production of $\mathrm{CDH}$ variants}

CDH variants for site-specific orientation on electrodes were recombinantly produced in Pichia pastoris and chromatographically purified to homogeneity (Table S2). Amounts of the variant enzymes between 21 and $333 \mathrm{mg}$ were obtained due to different cultivation volumes $(0.5-5 \mathrm{~L})$. SDS-PAGE showed for all variants similar molecular masses at about $100 \mathrm{kDa}$, which suggests a uniform glycosylation of the $\mathrm{CDH}$ variants (Figure S2). UV/Vis spectra showed that all enzymes exhibit the typical characteristics of a flavocytochrome with similar $\mathrm{R}_{\mathrm{Z}}$ values $\left(\mathrm{A}_{420} / \mathrm{A}_{280}\right)$ of 0.585 to 0.594 , indicating a uniform purity (Figure S3). To evaluate if the produced CDH variants also have similar catalytic and electron transfer rates in solution, which is a prerequisite of this study, the specific activity of the wild-type $\mathrm{CDH}$ and the seven $\mathrm{CDH}$ variants was determined for the substrate lactose and the electron acceptors 2,6-dichloroindophenol (DCIP) or cytochrome $c$ (Table S3). The expressed CDHs vary only slightly in catalytic lactose turnover, which was assessed by the DCIP assay (4.56 to $5.99 \mathrm{U} \mathrm{mg}^{-1}$ ). The reason can be minor differences in the FAD occupation of the active-site. ${ }^{23}$ The observed differences of the IET between DH and CYT measured with the cytochrome $c$ assay (1.27 to $1.98 \mathrm{U} \mathrm{mg}^{-1}$ ) vary to the same extent as the catalytic turnover and indicate that the introduced mutations exert no effect on the IET and CYT mobility. 


\section{Oriented immobilization of CDH variants on electrodes}

Covalent binding of CDH variants to a modified gold electrode was studied with a SPR instrument equipped with an electrochemical flow-cell. Free maleimide groups for enzyme immobilization were introduced onto the gold-sputtered disc as described in the Methods section (Supporting Information). To restrict DH to its assigned orientation, a short 3C-spacer (alanine) was used as linker between enzyme and electrode. The immobilization process of CDH variants was followed by SPR. The observed binding curves of all CDH variants were similar (Figure S4). The quick angle shift after the addition of a CDH variant in the flow-cell indicates a high concentration of $\mathrm{CDH}$ molecules close to the modified gold electrode, which initiates the binding process. The signal typically reaches $90 \%$ of its maximum intensity after $25 \mathrm{~s}$ and the electrode is fully covered after $100 \mathrm{~s}$. In the subsequent washing process the noncovalently bound CDH desorbs quickly, indicating a very low affinity of unspecifically absorbed CDH. This observation is supported by the same absorption behavior of wildtype $\mathrm{CDH}$ on the modified gold electrode which is completely removed by washing. The SPR angle shift from (a) to (c) in Figure S4 measures the amount of covalently bound $\mathrm{CDH}$ on the modified gold electrode. The specific surface coverage was calculated by using a conversion factor of $122 \mathrm{mdeg} \mathrm{ng}^{-1}$ of protein. The sitespecifically bound CDH variants had a surface coverage between $0.91-5.67 \mathrm{ng} \mathrm{mm}^{-2}$, typically around $2 \mathrm{ng} \mathrm{mm}^{-2}$ (Table S4). The high variability results from the individual preparation of each electrode, which is demonstrated by the differences between electrodes modified with the same $\mathrm{CDH}$ variant. The desorption of unspecifically bound $\mathrm{CDH}$ molecules by the washing step leaves the single layer of site-specifically immobilized CDH molecules with some variable space around them. SPR gold disc electrodes with immobilized CDH variants were tested for mediated electron transfer (MET) and DET by CV in $50 \mathrm{mM}$ Bis-Tris buffer, pH 5.5 with or without ferrocenemethanol as redox mediator. Figure S5 shows typical cyclic voltammograms of a CDH variant with and without substrate. The catalytic current of each $\mathrm{CDH}$ variant was calculated from the current difference at the anodic peak at $158 \mathrm{mV}$ vs. $\mathrm{Ag} \mid \mathrm{AgCl}$. The measured MET current correlates very well with the amount of absorbed enzyme and is on average $2.8 \mathrm{nA} \mathrm{ng}^{-1}$ (Table S4). The similar specific MET currents also demonstrate that the catalytic turnover in the $\mathrm{DH}$ domains of the $\mathrm{CDH}$ variants is not compromised by the different binding orientations. The specific current obtained with 
ferrocenemethanol can be recalculated into a turnover number of $2.6 \mathrm{~s}^{-1}$, which compares well to the reported turnover numbers for ferroceniumhexafluorophospahe $\left(1.6 \mathrm{~s}^{-1}\right)$, DCIP $\left(0.9 \mathrm{~s}^{-1}\right)$, and 1,4-benzoquinone $\left(3.8 \mathrm{~s}^{-1}\right)$ at $\mathrm{pH} 5.5 .{ }^{24}$ However, the DET current could be determined reproducibly only for two CDH variants, namely D553Cleft

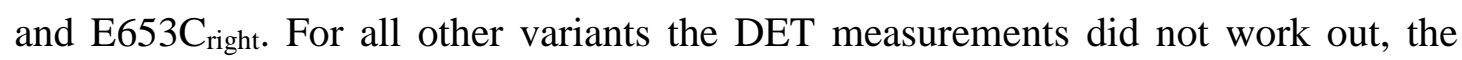
measurement error was higher than the observed current, or the obtained data were incoherent. Therefore, the DET currents of CDH variants were further tested on glassy carbon electrodes using a conventional three-electrode electrochemical cell.

\section{DET currents of $\mathrm{CDH}$ variants with anisotropic orientation}

To measure the DET currents of site-specific immobilized CDH variants reproducibly, we repeated the measurements in the SPR flow-cell, by exchanging the sputtered gold electrodes for glassy carbon electrodes which gave higher and much more reproducible DET currents for the immobilized CDH variants. To avoid an overload of the electrode surface with unspecifically and covalently bound $\mathrm{CDH}$ and to provide sufficient space around a $\mathrm{CDH}$ molecule for the movement of $\mathrm{DH}$ and $\mathrm{CYT}$ domains, the protein concentration in the immobilization process was reduced to $0.05 \mathrm{mg} \mathrm{mL}^{-1}$, which reduced the obtained DET per electrode to $~ 50 \%$ from the maximum current obtained for a full monolayer (Figure S6). Before measuring the DET and MET current in a thermostated electrochemical cell using a three-electrode setup, the physically adsorbed $\mathrm{CDH}$ was removed by stringent washing. The first series of measurements was performed at $\mathrm{pH}$ 5.5, where $M$. thermophilum $\mathrm{CDH}$ can form a closed conformation (CYT contacts DH). The closed conformation is important to allow for IET from DH to CYT. It has been shown that at a low $\mathrm{pH}$ electrostatic repulsion between both domains is reduced due to the protonation of surface exposed acidic amino acids. ${ }^{21}$ To account for different amounts of immobilized CDH, the MET current of each prepared electrode was measured and used to normalize its DET current. The side-on CDH variants D553Cleft and N561C left gave the highest normalized DET currents, followed by E653C right (Figure 2A). Interestingly, D553C left and E653C $C_{\text {right }}$ were the only two variants whose DET currents were also reliably detected on the modified gold electrodes. The DET currents of CDH variants immobilized on their back, bottom or top were 1.5 to 2 times lower. A preliminary interpretation could attribute this to the increased distance the CYT domain has to move between the closed conformation and the electrode surface. The lowest, 3 times smaller DET current was measured for 
E501C front, which can be interpreted by steric hindrance reducing the mobility of the CYT domain.

It has been reported that $M t C D H$ is not capable of IET at $\mathrm{pH} 7.5$, but that the addition of $30 \mathrm{mM} \mathrm{CaCl} 2$ can recover IET by relieving the electrostatic repulsion between $\mathrm{DH}$ and CYT. ${ }^{20,24}$ The higher MET currents obtained for all CDH variants indicate an 2.2 to 2.6-fold increased catalytic activity of $\mathrm{DH}$ at $\mathrm{pH}$ 7.5, which correlates with the reported increase in the FAD reduction rate in stopped-flow experiments. ${ }^{24}$ It also shows that the $\mathrm{CDH}$ orientation has no effect on the catalytic activity when using a soluble substrate and cosubstrate. However, also the measured DET currents in presence of $30 \mathrm{mM} \mathrm{CaCl}_{2}$ were generally higher than at pH 5.5 (Figure 2B) and indicate that in presence of $\mathrm{Ca}^{2+}$ the IET becomes fast even at this unsuitable, alkaline $\mathrm{pH}$. For the anisotropically oriented CDH variants (except D792C $\mathrm{C}_{\text {top }}$ and $\mathrm{E} 01 \mathrm{C}_{\text {front }}$ ) a 3.05 to 3.97 times increased DET current was measured, which corresponds to the trend observed for the heme reduction rate in the presence of $30 \mathrm{mM} \mathrm{CaCl}_{2}$ in stopped-flow experiments. $^{24}$ This correlation between heme reduction rate and DET current demonstrates that IET is the rate-limiting step in the electron transfer from $\mathrm{DH}$ to the electrode. Surprisingly, the DET currents for D792C $\mathrm{C}_{\text {top }}$ and $\mathrm{E} 01 \mathrm{C}_{\text {front }}$ increased in the presence of $30 \mathrm{mM} \mathrm{CaCl}_{2}$ even stronger (4.8 and 14.4 times, respectively) than for other variants. The presence of divalent cations supports the closed-state conformation of $\mathrm{CDH}$, which is not easily obtained in these two bound variants but necessary for a high IET rate.

\section{Correlation of orientation-dependent CDH mobility and DET}

The most surprising observation from Figure 2 is that DET was observed for all differently oriented CDH variants. This demonstrates that the CYT domain reaches far in its function as electron shuttle between the DH domain and the electron acceptor, which can be an electrode or the natural redox partner lytic polysaccharide monooxygenase (LPMO). ${ }^{25}$ The CDH variant's degree of mobility after orientationspecific immobilization is defined by all attainable inclination angles of an axis originating from the covalent point of attachment of the BOC- $\beta$-alanine linker to the DH's center of geometry (COG) with the electrode plane. The maximum angle of inclination was attained by rotating this axis from 90 degree up to the point when the first atom of the model came in contact with the virtual electrode plane. The inclination 
was determined for all possible rotations of the structural models of the $\mathrm{CDH}$ variants and DH domains around the indicated axis.

Some points of attachment bring the active-site channel of the DH domain closer to the electrode surface, which can result in two effects on DET. The positive effect of a closer distance is that in this position the CYT domain (in the closed conformation of CDH) is closer to the electrode surface, resulting in a shorter CYT domain movement to reach the electrode and perform DET. The negative effect of too close a distance is, that CYT may be positioned between $\mathrm{DH}$ and the electrode surface, which hinders CYT dissociation from DH into the open conformation, or, in the case that DH moves close to the electrode surface in the open conformation, that it prevents CYT by steric hindrance to attain its closed conformation. To evaluate the mobility of $\mathrm{CDH}$ and DH when covalently bound to a flat electrode surface, a rigid body approach was applied and polar plots were computed (Figure $\mathbf{3 A \& B}$ ).

A higher enclosed surface area in these figures reflects a higher degree of mobility of a $\mathrm{CDH}$ variant. The presence of the CYT domain in $\mathrm{CDH}$ reduced the maximum inclination angle of all variants except N351C back. This shows that steric hindrance in six of the variants can hinder CYT to a certain extent to move between the closed- or open conformation. The CYT of N351C back in CDH's closed conformation is located on the opposite side of the electrode, which prevents any contact with the electrode surface. The presence of the CYT domain in $\mathrm{D}$ 292 $\mathrm{C}_{\text {top }}$ and $\mathrm{N} 561 \mathrm{C}_{\text {left }}$ reduced the inclination angle of these variants, which have their CYT binding interface (at the substrate channel entrance of $\mathrm{CDH}$ ) relatively far away from the electrode surface. In contrast, the mobility of E501C front, $_{\text {D553C }}$ left, E653C right, and T680C $_{\text {bottom, which have }}$ their anchor point closer to the CYT binding interface showed a more restricted degree of mobility for CDH's closed conformation. The bar plot in Figure 3B gives the fraction of the maximal area obtained for each variant relative to the area of DH of

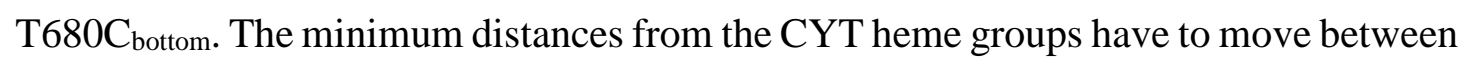
the DH interaction site and the electrode surface are shown in the box plots of Figure 3C for all the calculated rotational rigid models. The data generated for the polar plots was used to analyse the shortest path the heme proprionate A has to take from its contact point R698 in the closed conformation to the electrode surface. The arginine on the substrate channel entrance of DH forms an electrostatic interaction with the proprionate A group of the heme cofactor of CYT in the closed-state. ${ }^{15}$ 
How does the calculated domain mobility and CYT-electrode distance correlate with the measured DET current? By combining the previous observations we postulate that a small obstruction of the CYT mobility and a shorter CYT-electrode distance results in a faster DET (a higher DET:MET ratio). Since variant D792 $\mathrm{C}_{\mathrm{top}}$ mimics the natural orientation of the enzyme on cellulose, we expect that the enzyme has been evolved for good ET to its natural redox partner LPMO in this position. Indeed, the CYT mobility is almost unobstructed by the interaction with a surface and the CYT-electrode distance is one of the shortest. This is similar to the observation that a decreased distance between the electrode surface and the $\mathrm{T} 1 \mathrm{Cu}$ atom in bilirubin oxidase facilitated the interfacial electron transfer. ${ }^{26}$ This short distance results in only the fourth highest DET:MET ratio at $\mathrm{pH} 5.5$ and the third highest DET:MET ratio at $\mathrm{pH}$ 7.5. How important is the average CYT-electrode distance for this result? $\mathrm{CDH}$ variant

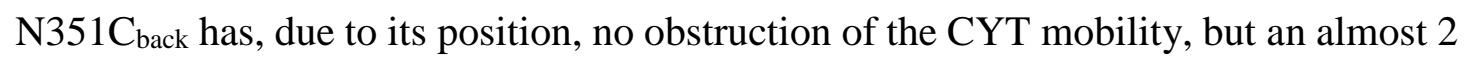
times longer CYT-electrode distance. The DET:MET ratio at $\mathrm{pH} 5.5$ is $10 \%$ lower and at $\mathrm{pH} 7.5$ is $36 \%$ lower than for $\mathrm{D} 792 \mathrm{C}_{\text {top }}$ in the natural orientation. What is the

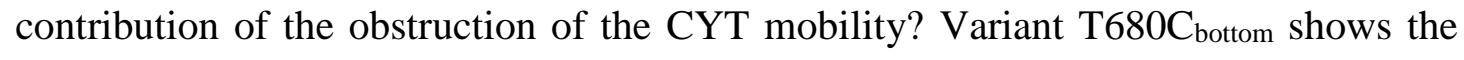
inverse orientation to $\mathrm{D} 72 \mathrm{C}_{\text {top. }}$. The CYT-electrode distance is similar, but the CYT mobility is reduced with about $40 \%$ of the possible rotations obstructed by the surface. The DET:MET ratio at $\mathrm{pH} 5.5$ is $19 \%$ lower and at $\mathrm{pH} 7.5$ is 35\% lower than that for D792C $C_{\text {top. }}$ For these three variants the hypothesis that an unobstructed CYT mobility and a shorter CYT-electrode distance result in a faster DET is confirmed.

The following four variants serve as test cases for this hypothesis. The most interesting change in DET:MET ratio is observed for E501C front. At pH 5.5 the normalized DET current is just half of that of $\mathrm{D}$ 92 $\mathrm{C}_{\text {top }}$, which comes partially from the slightly higher CYT-electrode distance, but also from the obstruction of the CYT mobility whose binding-site is close to the amino acid used for immobilization. In this orientation, the time-limiting step will be the association of CYT to DH from the open conformation, because it depends also on the movement of $\mathrm{DH}$ to generate the necessary distance to the electrode. However, at $\mathrm{pH} 7.5$ in the presence of $\mathrm{Ca}^{2+}$ the closed conformation is supported and the restricted mobility of DH results in limited motion and high CYT mobility leading to a dramatic increase in the DET:MET ratio, which is the highest of all variants. It is reported that a higher ionic strength also increases IET in sulfite oxidase by supporting the domain mobility. ${ }^{27}$ The limitation of the $\mathrm{DH}$ domain angular mobility together with $\mathrm{Ca}^{2+}$ as glue to support the closed conformation keeping the CYT domain close to DH, reduces the CYT traveling distances and decreases the time 
between open and closed conformations. The three other variants with restricted CYT

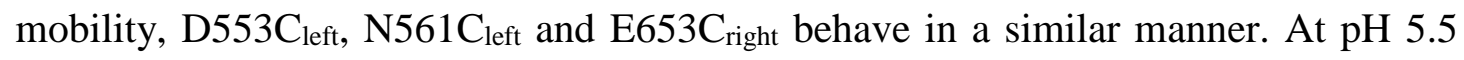
their DET:MET ratio is the highest of all variants. Variant E653C $\mathrm{C}_{\text {right }}$ has the lowest DET:MET ratio of these three, because of the biggest CYT-electrode distance. Why

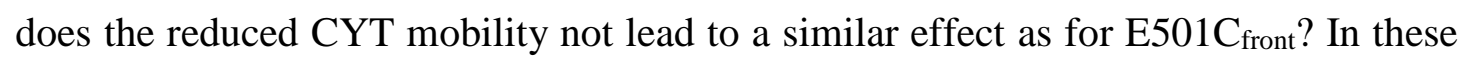
orientations the DH domain is less likely to completely block the access of CYT to its substrate channel/interaction site. The effect of the $\mathrm{Ca}^{2+}$ ions at $\mathrm{pH} 7.5$ is therefore also less pronounced, but the DET:MET ratios of D553C left, $\mathrm{N}_{61 \mathrm{C}_{\text {left }}}$ and $\mathrm{E} 653 \mathrm{C}_{\text {right }}$ are still the second, fourth and fifth highest, respectively.

\section{How the mobility of CYT rescues DET}

The mobility of CYT has been demonstrated above to be affected by the orientation of the DH domain, but it also depends on the length of the interdomain linker. In $M t C D H$ this linker is composed of 23 amino acids, mostly, Val, Gly and Thr resulting in a highly hydrophilic part of the enzyme. Therefore, in the crystal structure 4QI6 no defined structure of the linker region was observed. A short linker can prevent CYT from reaching the electrode, a longer interdomain linker long will not efficiently guide CYT back into the closed conformation. To explore how the interdomain linker length influences the CYT domain's ability to contact the electrode surface in the above simulated orientations of the variants, we used a MD simulation driven workflow to determine possible positions of CYT relative to $\mathrm{DH}$. We measured the conformational strain on the linker when positioning CYT at a large number of reference positions and orientations around DH (Figure S7). The resulting data were filtered based on two criteria: 1) A too high strain results in unfolding and termination of the simulation. In some of the successful simulations, primarily at longer distances between the two domains, a distortion of CYT was observed due to a high strain between the two domains. To reject these cases, a $0.25 \mathrm{~nm}$ backbone RMSD cut-off was applied to CYT; 2) the total restraining energies were considered to filter out MtCYT positions that have not reached their reference position. All reference positions relative to $\mathrm{DH}$ could be reached with the exception of the backside of CDH (Figure 4A, Figure S8). Very similar distributions are obtained when using a different energy cutoff. Hence it is reasonable to assume that accessible positions for CYT are limited to the area around the DH substrate channel - the CYT-DH interaction site - and the top, bottom, left and right sides around this front area. 


\section{Conclusions}

DET from CDH to the electrode surface is amazingly robust. The high flexibility and reach of the CYT domain in CDH's open conformation ensures DET in even the most awkward orientations. In this study, we observed with the generated seven variants not a single orientation of the immobilized $\mathrm{CDH}$, which prevented DET. The difference in the DET:MET ratio between the best and worst orientation depended on the $\mathrm{pH}$ and $\mathrm{Ca}^{2+}$ ion conditions, but was never bigger than a factor of three at the physiological $\mathrm{pH}$ optimum of $\mathrm{MtCDH}$ (5.5) or a factor of two under $\mathrm{Ca}^{2+}$ induced IET at $\mathrm{pH}$ 7.5. The positions found to have lower DET current were those, where the mobility of CYT to change between the closed and the open conformations was restricted and therefore limits IET. This study is in accordance with previous work, which found that for all studied cases DET was much faster than IET. ${ }^{19 b, 28}$ The optimum orientation of CDH on the electrode for a fast IET and DET is therefore on the side with a minimum traveling distance of CYT between its binding-site at the DH domain and the electrode surface. Additionally, a restricted mobility of CYT helps to minimize the extended stay in the open conformation with the CYT domain far removed from DH. On the other hand, a DH domain bound to the electrode surface with its interaction-site poorly accessible reduced the probability of CYT to reach the closed conformation. The high mobility of the CYT domain ensures both an efficient ET to LPMO and electrode surfaces. This ability of CYT is a great bonus for the enzyme's immobilization on bioelectrodes, since it does not necessitate oriented immobilization. The application of $\mathrm{CDH}$ on electrode surfaces resembles its natural function on cellulose microfibrils. There the CYT domain is evolved to transfer electrons to LPMO, which is also attached to polymer surfaces. The CYT of $\mathrm{CDH}$ is a universal electron shuttle, "a built-in mediator" 29 with a high flexibility. Since the IET is rate limiting, the optimization of the DET current should start with improving IET. Several other CDHs have higher IET rates compared to $\mathrm{MtCDH}$. Optimization of the CYT-DH interface and the linker length can improve IET and therefore DET, which would lead to higher currents in biofuel cells and a higher sensitivity in biosensors.

\section{Supporting Information}

The supporting information is available free of charge on the ACS Publications website at DOI: 
Supporting text, figures, tables and references for nucleotide sequences, enzyme purification and characterization, DET, MET and SPR measurements and CYT reference positions (PDF)

\section{Acknowledgements}

Funding is gratefully acknowledged from the European Commission (Project BIOENERGY FP7-PEOPLE-2013-ITN-607793), the European Union's Horizon 2020 research and innovation program (ERC Consolidator Grant OXIDISE) under grant agreement Nr. 726396 and the Austrian Science Fund (doctoral program BioToP, W1224). P.N.B. acknowledges receipt of a Wolfson Research Merit Award and L.G. a grant from the Swedish Research Council (2014-5908). 


\section{References}

(1) (a) Karyakin, A. A. Principles of Direct (Mediator Free) Bioelectrocatalysis. Bioelectrochemistry 2012, 88, 70-75. (b) Leger, C.; Bertrand, P. Direct Electrochemistry of Redox Enzymes as a Tool for Mechanistic Studies. Chem. Rev. 2008, 108, 2379-2438.

(2) Shleev, S.; Tkac, J.; Christenson, A.; Ruzgas, T.; Yaropolov, A. I.; Whittaker, J. W.; Gorton, L. Direct Electron Transfer between Copper-Containing Proteins and Electrodes. Biosens. Bioelectron. 2005, 20, 2517-2554.

(3) Grigorenko, V. G.; Andreeva, I. P.; Rubtsova, M. Y.; Egorov, A. M. Recombinant Horseradish Peroxidase: Production and Analytical Applications. Biochemistry (Mosc). 2015, 80, 408-416.

(4) Lubitz, W.; Ogata, H.; Rudiger, O.; Reijerse, E. Hydrogenases. Chem. Rev. 2014, 114, 4081-4148.

(5) Ma, S.; Ludwig, R. Direct Electron Transfer of Enzymes Facilitated by Cytochromes. Chemelectrochem 2019, 6, 958-975.

(6) Hernandez, K.; Fernandez-Lafuente, R. Control of Protein Immobilization: Coupling Immobilization and Site-Directed Mutagenesis to Improve Biocatalyst or Biosensor Performance. Enzyme Microb. Technol. 2011, 48, 107-122.

(7) Vaz-Dominguez, C.; Campuzano, S.; Rudiger, O.; Pita, M.; Gorbacheva, M.; Shleev, S.; Fernandez, V. M.; De Lacey, A. L. Laccase Electrode for Direct Electrocatalytic Reduction of $\mathrm{O}_{2}$ to $\mathrm{H}_{2} \mathrm{O}$ with High-Operational Stability and Resistance to Chloride Inhibition. Biosens. Bioelectron. 2008, 24, 531-537.

(8) Ulyanova, Y.; Babanova, S.; Pinchon, E.; Matanovic, I.; Singhal, S.; Atanassov, P. Effect of Enzymatic Orientation Through the Use of Syringaldazine Molecules on Multiple Multi-Copper Oxidase Enzymes. Phys. Chem. Chem. Phys. 2014, 16, 1336713375.

(9) Cazelles, R.; Lalaoui, N.; Hartmann, T.; Leimkuhler, S.; Wollenberger, U.; Antonietti, M.; Cosnier, S. Ready to Use Bioinformatics Analysis as a Tool to Predict Immobilisation Strategies for Protein Direct Electron Transfer (DET). Biosens. Bioelectron. 2016, 85, 90-95.

(10)(a) Ferapontova, E. E.; Ruzgas, T.; Gorton, L. Direct Electron Transfer of Hemeand Molybdopterin Cofactor-Containing Chicken Liver Sulfite Oxidase on Alkanethiol-Modified Gold Electrodes. Anal. Chem. 2003, 75, 4841-4850; (b) E. Ferapontova, E. ; Gorton, L. Direct Electrochemistry of Heme MulticofactorContaining Enzymes on Alkanethiol-Modified Gold Electrodes. Bioelectrochemistry 2005, 66, 55-63.

(11) (a) Xu, S.; Minteer, S. D. Investigating the Impact of Multi-Heme Pyrroloquinoline Quinone-Aldehyde Dehydrogenase Orientation on Direct Bioelectrocatalysis via Site Specific Enzyme Immobilization. Acs Catal. 2013, 3, 1756-1763; (b) Tsujimura, S.; Asahi, M.; Goda-Tsutsumi, M.; Shirai, O.; Kano, K.; Miyazaki, K. Direct Electron Transfer to a Metagenome-Derived Laccase Fused to Affinity Tags near the Electroactive Copper Site. Phys. Chem. Chem. Phys. 2013, 15, 20585-20589; (c) 
Campbell, W. H.; Henig, J.; Plumere, N. Affinity Binding via Zinc(Ii) for Controlled Orientation and Electrochemistry of Histidine-Tagged Nitrate Reductase in SelfAssembled Monolayers. Bioelectrochemistry 2013, 93, 46-50.

(12)Guan, D.; Kurra, Y.; Liu, W.; Chen, Z. A Click Chemistry Approach to SiteSpecific Immobilization of a Small Laccase Enables Efficient Direct Electron Transfer in a Biocathode. Chem. Commun. (Camb) 2015, 51, 2522-2525.

(13)(a) Kartashov, A. V.; Serafini, G.; Dong, M.; Shipovskov, S.; Gazaryan, I.; Besenbacher, F.; Ferapontova, E. E. Long-range Electron Transfer in Recombinant Peroxidases Anisotropically Orientated on Gold Electrodes. Phys. Chem. Chem. Phys. 2010, 12, 10098-10107; (b) Ferapontova, E.; Schmengler, K.; Borchers, T.; Ruzgas, T.; Gorton, L. Effect of Cysteine Mutations on Direct Electron Transfer of Horseradish Peroxidase on Gold. Biosens. Bioelectron. 2002, 17, 953-963.

(14)Lindgren, A.; Larsson, T.; Ruzgas, T.; Gorton, L. Direct Electron Transfer between the Heme of Cellobiose Dehydrogenase and Thiol Modified Gold Electrodes. J. Electroanal. Chem. 2000, 494, 105-113.

(15)Tan, T. C.; Kracher, D.; Gandini, R.; Sygmund, C.; Kittl, R.; Haltrich, D.; Hallberg, B. M.; Ludwig, R.; Divne, C. Structural Basis for Cellobiose Dehydrogenase Action during Oxidative Cellulose Degradation. Nat. Commun. 2015, 6, 7542.

(16)Ludwig, R.; Harreither, W.; Tasca, F.; Gorton, L. Cellobiose Dehydrogenase: a Versatile Catalyst for Electrochemical Applications. Chemphyschem 2010, 11, 26742697.

(17)(a) Kovacs, G.; Ortiz, R.; Coman, V.; Harreither, W.; Popescu, I. C.; Ludwig, R.; Gorton, L. Influence of SAM Structure on Direct Electron Transfer at Au Electrodes Modified with Cellobiose Dehydrogenase from Neurospora Crassa. Rev. Roum. Chim. 2012, 57, 361-368; (b) Sarauli, D.; Ludwig, R.; Haltrich, D.; Gorton, L.; Lisdat, F. Investigation of the Mediated Electron Transfer Mechanism of Cellobiose Dehydrogenase at Cytochrome C-Modified Gold Electrodes. Bioelectrochemistry 2012, 87, 9-14; (c) Matsumura, H.; Ortiz, R.; Ludwig, R.; Igarashi, K.; Samejima, M.; Gorton, L. Direct electrochemistry of Phanerochaete chrysosporium cellobiose dehydrogenase covalently attached onto gold nanoparticle modified solid gold electrodes. Langmuir 2012, 28, 10925-10933; (d) Wang, X.; Falk, M.; Ortiz, R.; Matsumura, H.; Bobacka, J.; Ludwig, R.; Bergelin, M.; Gorton, L.; Shleev, S. Mediatorless Sugar/Oxygen Enzymatic Fuel Cells Based on Gold Nanoparticle-Modified Electrodes. Biosens. Bioelectron. 2012, 31, 219-225.

(18)(a) Tasca, F.; Harreither, W.; Ludwig, R.; Gooding, J. J.; Gorton, L. Cellobiose Dehydrogenase Aryl Diazoniunn Modified Single Walled Carbon Nanotubes: Enhanced Direct Electron Transfer through a Positively Charged Surface. Anal. Chem. 2011, 83, 3042-3049; (b) Ortiz, R.; Ludwig, R.; Gorton, L. Highly Efficient Membraneless Glucose Bioanode Based on Corynascus thermophilus Cellobiose Dehydrogenase on Aryl Diazonium-Activated Single-Walled Carbon Nanotubes. Chemelectrochem 2014, 1, 1948-1956. 
(19)(a) Al-Lolage, F. A.; Meneghello, M.; Ma, S.; Ludwig, R.; Bartlett, P. N. A Flexible Method for the Stable, Covalent Immobilization of Enzymes at Electrode Surfaces. ChemElectroChem 2017, 4, 1528-1534; (b) Meneghello, M.; Al-Lolage, F. A.; Ma, S.; Ludwig, R.; Bartlett, P. N. Studying Direct Electron Transfer by Site-Directed Immobilization of Cellobiose Dehydrogenase. Chemelectrochem 2019, 6, 700-713.

(20) Schulz, C.; Ludwig, R.; Micheelsen, P. O.; Silow, M.; Toscano, M. D.; Gorton, L. Enhancement of Enzymatic Activity and Catalytic Current of Cellobiose Dehydrogenase by Calcium Ions. Electrochem. Commun. 2012, 17, 71-74.

(21)(a) Kadek, A.; Kavan, D.; Felice, A. K.; Ludwig, R.; Halada, P.; Man, P. Structural Insight into the Calcium Ion Modulated Interdomain Electron Transfer in Cellobiose Dehydrogenase. FEBS Lett. 2015, 589, 1194-1199; (b) Kadek, A.; Kavan, D.; Marcoux, J.; Stojko, J.; Felice, A. K.; Cianferani, S.; Ludwig, R.; Halada, P.; Man, P. Interdomain Electron Transfer in Cellobiose Dehydrogenase is Governed by Surface Electrostatics. Biochim. Biophys. Acta 2016, 1861, 157-167.

(22)Bodenheimer, A. M.; Odell, W. B.; Oliver, R. C.; Qian, S.; Stanley, C. B.; Meilleur, F. Structural Investigation of Cellobiose Dehydrogenase IIA: Insights from Small Angle Scattering into Intra- and Intermolecular Electron Transfer Mechanisms. BbaGen Subjects 2018, 1862, 1031-1039.

(23)Ma, S.; Preims, M.; Piumi, F.; Kappel, L.; Seiboth, B.; Record, E.; Kracher, D.; Ludwig, R. Molecular and Catalytic Properties of Fungal Extracellular Cellobiose Dehydrogenase Produced in Prokaryotic and Eukaryotic Expression Systems. Microb. Cell Fact. 2017, 16, 37.

(24)Kracher, D.; Zahma, K.; Schulz, C.; Sygmund, C.; Gorton, L.; Ludwig, R. InterDomain Electron Transfer in Cellobiose Dehydrogenase: Modulation by $\mathrm{pH}$ and Divalent Cations. Febs. J. 2015, 282, 3136-3148.

(25)Kracher, D.; Scheiblbrandner, S.; Felice, A. K.; Breslmayr, E.; Preims, M.; Ludwicka, K.; Haltrich, D.; Eijsink, V. G.; Ludwig, R. Extracellular Electron Transfer Systems Fuel Cellulose Oxidative Degradation. Science 2016, 352, 1098-1101.

(26) Matanovic, I.; Babanova, S.; Chavez, M. S.; Atanassov, P. Protein-Support Interactions for Rationally Designed Bilirubin Oxidase Based Cathode: A Computational Study. J. Phys. Chem. B 2016, 120, 3634-3641.

(27)Utesch, T.; Sezer, M.; Weidinger, I. M.; Mroginski, M. A. Adsorption of Sulfite Oxidase on Self-Assembled Monolayers from Molecular Dynamics Simulations. Langmuir 2012, 28, 5761-5769.

(28) Matsumura, H.; Ortiz, R.; Ludwig, R.; Igarashi, K.; Samejima, M.; Gorton, L. Direct Electrochemistry of Phanerochaete chrysosporium Cellobiose Dehydrogenase Covalently Attached onto Gold Nanoparticle Modified Solid Gold Electrodes. Langmuir 2012, 28, 10925-10933.

(29)Ikeda, T.; Kobayashi, D.; Matsushita, F.; Sagara, T.; Niki, K. Bioelectrocatalysis at Electrodes Coated with Alcohol-Dehydrogenase, a Quinohemoprotein with Heme- $c$ Serving as a Built-in Mediator. J. Electroanal. Chem. 1993, 361, 221-228. 


\section{Figure Captions}

Figure 1. Overview of the individual anchor residue introduced to seven, single CDH variant. DH domain (blue), FAD (yellow), CYT domain (orange), heme (red), linker region (cyan), CBM (green). The fold of the $M$. thermophilum CDH crystal structure 4QI6 is shown in ribbon representation, the cofactors in stick representation. The single mutations at N351 (blue), E501 (red), T680 (orange) and D792 (green) are shown in the left panel (A), while the mutations at D553 (light cyan), N561 (cyan) and E653 (purple) are shown in the right panel (B). The mutated residues are represented by spheres, their position in the protein sequence and in regard to the electrode is indicated in the accompanying table.

Figure 2. Comparison of the DET:MET ratios of $\mathrm{CDH}$ variants at $\mathrm{pH} 5.5$ (A) and $\mathrm{pH}$ 7.5 in the presence of $30 \mathrm{mM} \mathrm{CaCl}_{2}$ (B). The mean values of three replicates of the specific DET and MET currents and the DET:MET rations are given in the table.

Figure 3. $M t \mathrm{CDH}$ vs $M t \mathrm{DH}$ mobility on a flat surface. (A) Polar plots showing the mobility of $M t \mathrm{DH}$ (grey area) and $M t \mathrm{CDH}$ (PDB ID: 4QI6, closed CDH conformation, red hatched area) when attached to a flat surface with a linker. The $\mathrm{C}_{\alpha}$ atoms of the respective anchor residue and the $M t D H C O G$ were aligned with the z-axis. The angular axis of the polar plots shows the rotation of $M t \mathrm{DH}$ and $M t \mathrm{CDH}$ around the $\mathrm{Z}$-axis. The radial axis shows the maximum tilt angle. A high value for the maximum tilt angle translates into a high mobility of $\mathrm{DH}$ or $\mathrm{CDH}$. (B) The bar plot gives the areas enclosed by the polar plot curves as fractions of the maximum area relative to $\mathrm{DH}$ of T680Cbottom. A large number of high tilt angles correspond to a high area and a higher mobility of the DH and $\mathrm{CDH}$. (C) Boxplots showing the calculated minimum distances of the Arg698 CZ atom to the electrode surface without going through the protein. 
Figure 4. (A) Distribution of $M t C Y T$ around $M t D H$ after $M t C Y T$ sampling and filtering. $M t D H$ and the CBM are shown in orange and green respectively. MtCYT COG distributions are blown up to the MtCYT radius of gyration $(1.6 \mathrm{~nm})$. All resulting models have been filtered according to a $0.25 \mathrm{~nm}$ RMSD cut-off considering the MtCYT backbone atoms and based on the total restraining energy term (max $2 \mathrm{MJ} \mathrm{mol}^{-}$

${ }^{1}$ ). (B) Number of possible $M t C Y T$ positions around $M t D H$ at different total position restraints energies after filtering with a $0.25 \mathrm{~nm}$ MtCYT backbone RMSD cut-off. 
TOC Graphic
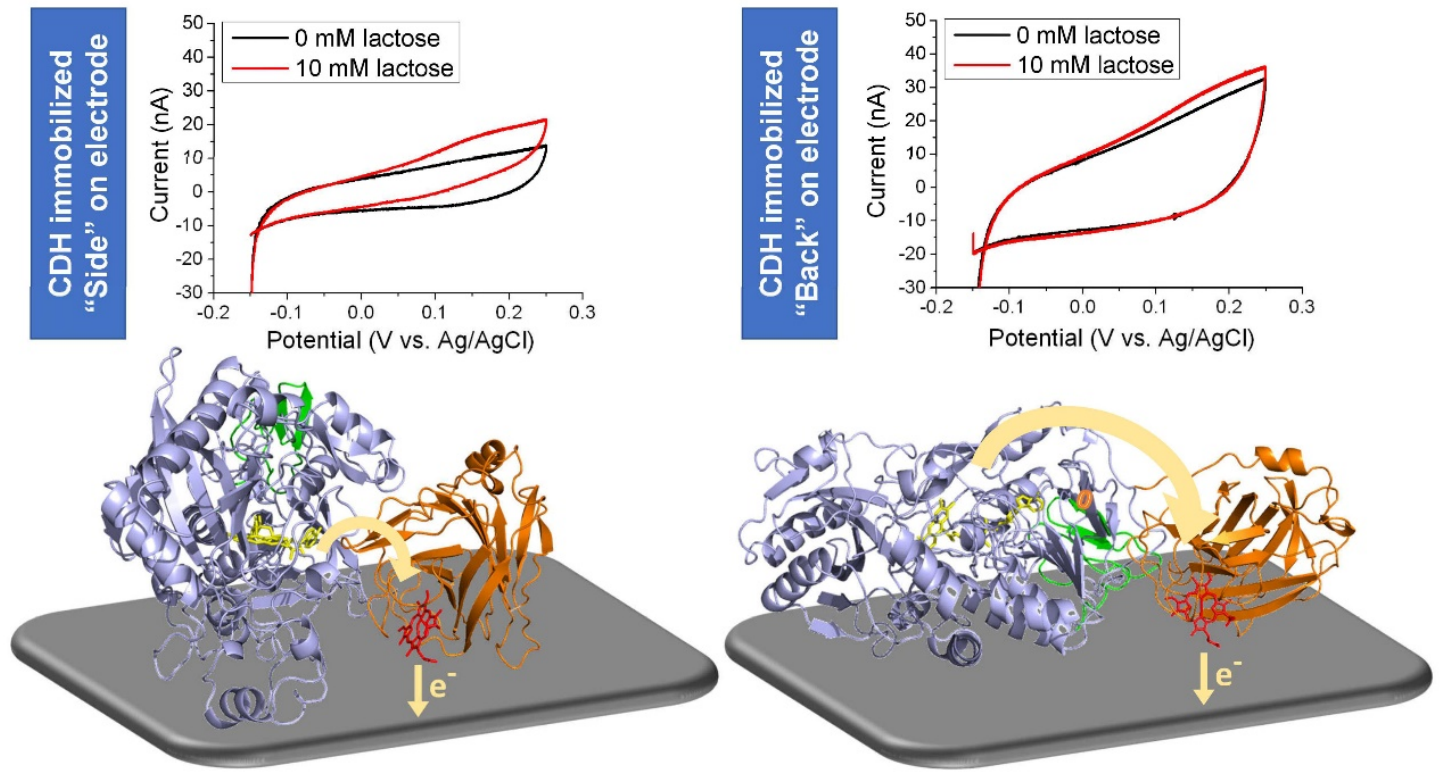